\title{
THE VALIDATION OF THE MASLACH BURNOUT INVENTORY - HUMAN SERVICES SURVEY FOR EMERGENCY MEDICAL TECHNICIANS IN GAUTENG*
}

\author{
J.L.P. NAUDÉ \\ S. ROTHMANN \\ WorkWell: Research Unit for People, Policy and Performance, \\ Faculty of Economic and Management Sciences, \\ North-West University, Potchefstroom
}

\begin{abstract}
The objectives of this study were to validate the Maslach Burnout Inventory - Human Services Survey (MBI-HSS) for emergency medical technicians in the Gauteng Province of South Africa and to determine its construct equivalence and bias for different race groups. A cross-sectional survey design with an accidental sample $(N=318)$ was used. The MBIHSS and a biographical questionnaire were administered. Evidence of uniform bias was found for one item of the MBIHSS. Exploratory factor analyses resulted in a 3-factor model of burnout, consisting of Emotional Exhaustion, Depersonalisation and Personal Accomplishment. The scales showed acceptable internal consistencies. Exploratory factor analysis with target rotations confirmed construct equivalence of scales for the White and Black groups.
\end{abstract}

\section{OPSOMMING}

Die doelstellings van hierdie studie was om die Maslach Uitbrandingsvraelys - Menslike Dienste-Opname (MBI-HSS) te valideer vir die Nood Mediese Tegnici in die Gauteng Provinsie van Suid-Afrika en om die konstrukekwivalensie en sydigheid daarvan vir die verskillende rassegroepe te bepaal. 'n Dwarssnee opname-ontwerp met 'n beskikbaarheidsteekproef $(N=318)$ is gebruik. Die MBI-HSS en 'n biografiese vraelys is afgeneem. Uniforme sydigheid is gevind vir een item van die MBI-HSS. Verkennende faktorontleding met teikenrotasies het geresulteer in 'n 3-faktormodel van uitbranding bestaande uit Emosionele Uitputting, Depersonalisasie en Persoonlike Bereiking. Die skale het aanvaarbare interne konsekwentheid getoon. Verkennende faktorontleding met teikenrotasies het die konstrukekwivalensie vir die drie faktore bevestig vir die Wit en Swart groepe.

Emergency medical technicians are often confronted with extremely stressful and demanding situations. According to Vettor and Kosinski (2000), emergency medical technicians constantly face chronic stressors, such as having to deal with injury, mutilation and even death. Frank and Ovens (2002) point to the fact that emergency work is both rewarding and demanding in that little control over patient-mix exists, compounded by the fact that life or death decisions have to be dealt with at a rapid pace. Many of these situations are difficult to manage, often resulting in psychological distress for the emergency worker who functions in a chronic stress environment where stress may be perceived as part of the job (Phipps, 1988; Whitley, Gallery, Allison \& Revicki, 1989; Young $\&$ Cooper, 1995). The impact of highly stressful environments on the emergency medical technicians is tremendous, often resulting in an increased risk of injury, cardiovascular disease and other health problems, psychological health disorders and burnout (Kowalski \& Vaught, 2001).

Burnout can be described as a specific type of job stress which influences job-related affective well-being (Schaufeli \& Buunk, 2002). Although mainly psychological in nature, the prevalence of physical symptoms is not uncommon. More specifically, burnout affects the individual on a cognitive, affective, physical and behavioural level as a result of a general breakdown in defences against prolonged job-stress (Brill, 1984). This breakdown occurs gradually and often remains unnoticed for a long time, perpetuated by ineffective coping strategies and frustrated intentions brought about by the subjective experience of work-reality. The gradual depletion of emotional resources results in a sense of reduced effectiveness, decreased motivation and the development of dysfunctional behaviours and attitudes at work (Schaufeli \& Enzmann, 1998). According to these authors, burnout can be defined as a persistent, negative work-related state of mind in "normal" individuals, primarily characterised by emotional exhaustion

Requests for copies should be addressed to: S Rothmann, Department of Industrial Psychology, North-West University, Potchefstroom Campus, Private Bag X6001, Potchefstroom, 2520

* This material is based upon work supported by the National Research Foundation under Grant number 2053344 and accompanied by distress, a sense of reduced effectiveness, decreased motivation, and the development of dysfunctional attitudes and behaviours at work.

Originally restricted to the helping professions, burnout is now recognised as a phenomenon found in a variety of occupational groups (Cordes \& Dougherty, 1993). Research over the last three decades has shown that the consequences of burnout are not just limited to the individual's subjective experience, but also to various organisational outcomes. Burnout has been associated with reduced organisational efficiency and work-related problems such as employee turnover, low morale, poor quality of care, lowered productivity, absenteeism and interpersonal problems (Levert, Lucas \& Ortlepp, 2000; Rosse, Boss, Johnson \& Crown, 1991). Also, burnout has been associated with insomnia, perceptions of physical exhaustion and increased substance abuse (Jackson \& Maslach, 1982; Maslach, 1982). The study of burnout, therefore, certainly seems to be beneficial to the general welfare of companies and their workers in various organisational contexts.

Probably the most influential development in terms of scientific exploration of the burnout construct was the development of the Maslach Burnout Inventory (MBI) (Maslach \& Jackson, 1986). Three versions of the MBI were developed, namely the MBI-GS (General Survey), MBI-ED (Educators) and MBI-HSS (Human Services Survey). Whereas the MBI-GS measures Exhaustion, Cynicism and Professional Efficacy, the latter two measures Emotional Exhaustion, Depersonalisation and Personal Accomplishment. The General Survey measures burnout in a broad range of professions, whereas the HSS and ED-versions of the MBI measures burnout in the human services and education contexts respectively. Recently, Schaufeli, Martinez, Pinto, Salanova and Bakker (2002) developed a student version of the MBI. In the current study, however, the MBI-HSS is used, but to date, no validation studies on the MBI - HSS on the South African emergency worker context could be found. 
The importance of establishing a reliable and valid instrument to assess burnout in the emergency worker setting is not only important for empirical research purposes, but also for the pragmatic, standardised application in the individual assessment setting. As such, a considerable amount of research seems to support the psychometrical soundness of the MBI-HSS in various occupational settings (Byrne, 1991, 1994; Enzmann, Schaufeli \& Girault, 1994; Green \& Walkey, 1988; Maslach \& Jackson, 1981).

In terms of the validity of the MBI-HSS, there seems to be sufficient evidence for the convergent validity of the scale, but some difference of opinion exists regarding the discriminant validity of the MBI-HSS (Schaufeli \& Van Dierendonck, 1995). The convergent validity, established by gathering data in different ways to demonstrate convergence on the same construct, was established by the expected relationships of correlations of MBI-HSS scores with independent behavioural ratings of significant others, job characteristics expected to contribute to burnout and measures of various outcomes hypothesised to be related to burnout (Maslach, Jackson \& Leiter, 1997). In order to differentiate the MBI-HSS from other constructs and explain why they are/are not related to the construct of burnout, relationships between burnout and concepts such as job satisfaction, depression and social desirability were investigated. Small but significant positive relationships were found between different measures of satisfaction and the MBI-HSS scales (Maslach, Jackson \& Leiter, 1997).

In terms of South African studies, there seems to be an apparent paucity of research regarding the reliability and validity of the MBI-HSS. Although many related studies were conducted in the United States and Europe in the early stages of scale development, a lack of research in this area within the South African context necessitates the current research. Rothmann (2003) stressed the need for burnout research in South Africa in stating that serious limitations of burnout research in South Africa include poorly designed studies (i.e. small sample sizes), a lack of sophisticated statistical analyses and poorly controlled studies.

South Africa has a multicultural society. According to Van de Vijver and Leung (1997), measurement equivalence and bias should be computed for measuring instruments in any multicultural setting where individuals from different cultural groups are compared in terms of a specific construct. This is particularly relevant where no norms exist for the different cultural groups, which is often the case in cross-cultural research. In line with recommendations of Van de Vijver and Leung (1997) measurement equivalence and bias should be tested for in a multi-cultural context where differences in scores could be attributed to cultural influences in terms of item meaning and understanding, rather than resulting from differences in the underlying latent variable. If cultural influences are not accounted for, invalid conclusions regarding the constructs under study could be made with serious implications for culturally diverse settings such as South Africa. Where measurement equivalence is concerned with measurement and the comparability of scores, bias is concerned with factors that influence the validity of cross-cultural comparisons.

The objectives of this study were to determine the construct equivalence, item bias, factorial validity and internal consistency of the Maslach Burnout Inventory - Human Services Survey (MBI-HSS) for emergency medical technicians in Gauteng.

The Maslach Burnout Inventory - Human Services Survey A major development in the stimulation of scientific interest in burnout was the introduction of the easy to administer self-report questionnaires in the beginning of the eighties, the Maslach Burnout Inventory (MBI) in particular being the most popular (Maslach et al., 1996). It is estimated that the MBI was used in over $90 \%$ of the empirical publications on burnout since the mid eighties (Schaufeli \& Enzmann, 1998). Koeske and Koeske (1993) state that the MBI has taken a central point in shaping the theoretical debate over the nature of burnout.

The MBI-HSS (Human Services Survey) was designed to measure burnout of people working in the human services and health care occupations by means of three subscales, namely Emotional Exhaustion, Depersonalisation and Personal Accomplishment. Emotional Exhaustion refers to a lack of energy and a feeling that emotional resources are depleted, whereas Depersonalisation refers to the treatment of recipients of services in a negative, cynical, detached and emotionally callous manner. Reduced Personal Accomplishment refers to negative selfevaluation, the belief that objectives are not reached, poor professional self-esteem and beliefs of insufficiency on the part of the service provider.

Initial interest in the first phase of development of the construct was limited to pragmatic and descriptive concerns and a lack of empirical study. It was only during the so-called empirical phase that the construct of burnout was operationally defined and researched by means of scientific study. There seems to be disagreement in the literature with regards to the factorial structure of the MBI-HSS (Cordes \& Dougherty, 1993, Maslach \& Jackson, 1986). Findings have indicated two-factor solutions (Brookings, Bolton, Brown \& McEvoy, 1985), four-factor solutions (Firth, McIntee, McKeown \& Britton, 1985; Iwanicki \& Schwab, 1981) and unitary conceptions of the factor structure of the MBIHSS, adding the three factors together towards an overall measure of burnout (Golembiewski \& Munzenrider, 1981).

In their sample of female human services professionals, Brookings et al. (1985) found a combined factor consisting of Emotional Exhaustion and Depersonalisation which they called the core of burnout, and a Personal Accomplishment factor. According to Firth et al. (1985), Emotional Exhaustion could be subdivided into two factors, namely Frustration and Discouragement about work and Emotional Draining in a nursing sample, whereas Iwanicki and Schwab (1981) argued for the separation of Depersonalisation into a job-related and student-related factor in their teacher sample. However, the most common solution in terms of the factorial structure of the MBI seems to be the existence of three conceptually distinct components of burnout (Golembiewski \& Munzenrider, 1981; Green \& Walkey, 1988; Maslach \& Jackson, 1981, 1986).

In terms of reliability, the MBI-HSS seems to be an internally consistent scale with Cronbach alphas in various samples (e.g graduate students, administrators in a health agency, teachers, social service and mental health workers, police officers, nurses and public service employees) constantly exceeding the proposed criterion of 0,70 proposed by Nunnally and Bernstein (1994) with the exception of the Depersonalisation scale in some samples (Schaufeli, Bakker, Hoogduin, Schaap \& Kladler, 2001; Schaufeli, Enzmann \& Girault, 1993). Initial research on the MBI-HSS yielded reliability coefficients of 0,90 for Emotional Exhaustion, 0,79 for Depersonalisation and 0,71 for Personal Accomplishment (Maslach et al., 1997). In a study of educators and business owners in the United States of America, Boles, Dean, Ricks, Short and Wang (2000) found Cronbach alpha coefficients of 0,89 and 0,90 (Emotional Exhaustion), 0,70 and 0,80 (Depersonalisation) and 0,76 and 0,78 (Personal Accomplishment) for the educators and small business owners respectively.

In the South African context, research evidence seems to confirm these findings. Basson and Rothmann (2002) found internal consistencies of 0,67 (Depersonalisation); 0,73 (Personal Accomplishment) and 0,89 for Emotional Exhaustion in a pharmacist sample. In their sample of psychiatric nurses, Levert et al. (2000) reported alpha coefficients of 0,74 (Depersonalisation); 0,75 (Personal Accomplishment) and 0,78 (Emotional Exhaustion).

Studies on the test-retest reliability of the MBI-HSS seem to confirm the stability of the MBI-HSS scales over time. Coefficients 
of 0,82 for Emotional Exhaustion, 0,60 for Depersonalisation and 0,80 for Personal Accomplishment was found after 2 to 4 weeks for a sample of social welfare graduate students and health agency administrators. Although the coefficients were low to moderately high, they were found statistically significant at the 0,001 level (Maslach et al., 1997). Another study amongst teachers $(n=248)$ revealed coefficients of 0,60 for Emotional Exhaustion, 0,54 for Depersonalisation and 0,57 for Personal Accomplishment after one year (Jackson, Schwab \& Schuler, 1986). Other studies confirmed stability of the scales of the MBI-HSS over time with correlations of 0,50 to 0,82 for time spans of three months to one year (Leiter \& Durup, 1996).

Meta-analysis in a study by Lee and Ashforth (1996) established the intercorrelation between the Emotional Exhaustion scale and the Depersonalisation scale at 0,64. The relationship between Emotional Exhaustion and Personal Accomplishment was determined at $-0,22$, while Depersonalisation and Personal Accomplishment yielded a correlation of $-0,34$. Notwithstanding this fact, differential patterns of correlations between the dimensions of burnout and other study variables such as age, workload, autonomy, job challenge, satisfaction with status and recognition, role ambiguity, job satisfaction, turnover intention, role conflict and organisational commitment, to name but a few, seem to suggest the existence of the three distinct components of burnout (Friesen, Prokop \& Sarros, 1988; Jackson et al., 1986; Maslach \& Jackson, 1984; Lee \& Ashforth, 1996; Schwab \& Iwanicki, 1982). This argument was also posited by Maslach in arguing against the existence of a single unitary conception of burnout (Iwanicki \& Schwab, 1981; Maslach \& Jackson, 1981).

In the last decades, statistical techniques have been developed that can cater for partial dissimilarity of items and factor structures. If there is a common set of items that measure the same latent trait in each culture group, for example analysis of variance (yielding interval-level scores), it allows for crosscultural comparison of item and person parameters. Similarly, scale-level analysis (construct equivalence) in the form of exploratory factor analysis can be used to test the equality of the factor structure even when not all the stimuli are identical across groups (cf. Muthén, 1991, 1994).

Construct equivalence (also known as structural equivalence) indicates the extent to which the same construct is measured across the cultural groups under study. In the case of structural inequivalence, no comparison can be made because scores obtained are not related to the same construct. Item bias, the second important computation in cross-cultural settings, concerns aspects of measurement validity in intercultural group comparisons (Van de Vijver \& Leung, 1997). An unbiased item would provide the same average score on an item if two people from different cultural groups are similar in terms of the construct measured by this item. Stated differently, individuals with an equal standing in terms of the underlying construct measured by the instrument would obtain the same score on a given item, irrespective of group membership. This does not, however, imply that the averages of the cultural groups must be exactly the same, but only that those individuals who are in reality equal in terms of their standing on the construct under study, should in fact obtain the same average score on the given item even though they differ in terms of group membership. In reality, differences in group averages occur, but these differences could be ascribed either to bias or legitimate differences between cultures, also known as impact.

Bias can be caused by incidental differences in appropriateness of item content, inadequate item formulation and translation, but also from response characteristics of the sample and administration effects. The danger associated with bias is that it would lower the equivalence of the measuring instrument. Two types of bias can be distinguished, namely uniform and non- uniform bias (Van de Vijver \& Leung, 1997). Uniform bias refers to the main effects of cultural differences, in other words the influence of bias on an item is consistent for all the score levels of that particular item. Non-uniform bias refers to the interaction effects of cultural differences and score level, indicating that across all score levels of an item, significantly larger differences in terms of a particular item exists in one group when compared to the other group across the different score levels for the specific item (Mellenbergh, 1982).

Storm and Rothmann (2003) investigated the construct equivalence and item bias of the MBI-GS for different race groups in the South African Police Service. They found construct equivalence for the MBI-GS, while no evidence of item bias could be found. However, no studies regarding the construct equivalence and item bias of the MBI-HSS were found.

The hypotheses of this study are as follows:

H1: Burnout, as measured by the MBI-HSS, can be defined as a three-dimensional construct with acceptable levels of internal consistency for each of its subscales, namely Emotional Exhaustion, Depersonalisation and Personal Accomplishment.

H2: The MBI-HSS is a construct equivalent and unbiased measuring instrument for the different race groups of emergency medical technicians in Gauteng.

\section{METHOD}

\section{Research design}

A cross-sectional survey design was used. Cross-sectional designs are appropriate where groups of subjects at various stages of development are studied simultaneously, whereas the survey technique of data collection gathers information from the target population by means of questionnaires (Shaughnessy \& Zechmeister, 1997).

\section{Study population}

An accidental sample of emergency medical technicians in the different regions of Gauteng, namely West Rand, Ekurhuleni, Sedibeng, Johannesburg Metropolitan, Tshwane, Kungwini and Nokeng Tsa Taemane was taken. The total population of 2100 emergency medical technicians in Gauteng was targeted. A response rate of $21,6 \%$ was achieved due to the nature of the job, e.g. call-outs, rotating working schedules and leave. Descriptive information of the sample is given in Table 1.

TABLE 1

Characteristics OF THE PARTICIPANTS

\begin{tabular}{llc}
\hline Item & Category & Percentage \\
\hline Home Language & Afrikaans & 45,00 \\
& English & 19,80 \\
& African & 35,20 \\
Position & Management & 16,70 \\
& Medical Specialists & 5,00 \\
& Emergency Medical Technicians & 72,00 \\
& Support Services & 6,30 \\
Area & West Rand & 11,90 \\
& Ekurhuleni & 45,90 \\
& Sedibeng & 17,00 \\
& Johannesburg Metro & 9,40 \\
& Tshwane & 8,20 \\
& Kungwini & 4,70 \\
Gender & Nokeng Tsa Taemane & 2,80 \\
& & \\
& Male & 77,72 \\
& Female & 22,28 \\
\hline
\end{tabular}


The sample consisted mainly of Afrikaans-speaking, married men $(58,4 \%)$ with a tertiary education (diploma). The mean age of the participants was 33,13 years while the average length of service was 9,68 years. Only $11,88 \%$ of the sample had a qualification lower than Grade 12. A total of $64,8 \%$ of the participants were White, while $35,2 \%$ were Black.

\section{Measuring battery}

The Maslach Burnout Inventory - Human Services Survey (MBIHSS) (Maslach \& Jackson, 1986; Maslach, Jackson \& Leiter, 1996) was used in this study. Also, biographical information was gathered regarding race, language, position, education, gender and marital status.

The Maslach Burnout Inventory - Human Services Survey (MBI-HSS) (Maslach \& Jackson, 1986; Maslach, Jackson \& Leiter, 1996) measures respondents' perceived experience of burnout in relation to the recipients of their service, care or treatment. The MBI-HSS consists of 22 items phrased as statements about personal feelings and attitudes, which is self-scored on a seven-point frequency scale, ranging from 0 (never) to 6 (every day). The three subscales of the MBI-HSS include Emotional Exhaustion (nine items; e.g. "I feel emotionally drained from my work"), Depersonalisation (five items; e.g. "I feel I treat some recipients as if they were impersonal objects"), and Personal Accomplishment (eight items; e.g. "I have accomplished many worthwhile things in this job"). High scores on Emotional Exhaustion and Depersonalisation and low scores on Personal Accomplishment are indicative of burnout. The subscales represent a related (Emotional Exhaustion and Depersonalisation) and independent (Personal Accomplishment), but separate multidimensional concept of the burnout construct. As such, the psychometric soundness of the MBI-HSS is well documented in the literature with internal consistencies usually well above the 0,70 Cronbach alpha level, except for the Depersonalisation scale in some samples (Schaufeli et al., 2001). Test-retest reliability ranging from three months to one year has been reported in the range of 0,50 to 0,82 (Leiter \& Durup, 1996).

\section{Statistical analysis}

The statistical analysis was carried out with the SPSS program (SPSS Inc., 2003). In the first step, means, standard deviations, skewness and kurtosis were determined to describe the data. The reliability and validity of the MBI-HSS were determined by means of Cronbach alpha coefficients, as well as exploratory factor analyses.

Item level analysis (item bias analysis) was performed by using analysis of variance (ANOVA) for the MBI-HSS (yielding intervallevel scores). The assumption is that an item is unbiased if persons from different race groups with an equal standing on the theoretical construct underlying the instrument have the same expected score on the item (e.g., Van de Vijver \& Leung, 1997). Although several statistical techniques are available for analysing item bias analysis of variance, it has the advantage of computational simplicity, robustness and the possibility to study both uniform and non-uniform bias (Mellenbergh, 1982). Therefore, analysis of variance was used in the present study. The item score was the dependent variable, while race and score levels were the independent variables. A significant main effect of race group was taken to point to uniform bias and a significant interaction of score level and interaction pointed to non-uniform bias.

Construct equivalence of the MBI-HSS was also assessed. Construct equivalence can be investigated with several techniques, such as factor analysis, cluster analysis, and multidimensional scaling or other dimensionality-reducing techniques (Van de Vijver \& Leung, 1997). The basic idea behind the application of these techniques is to obtain a structure in each culture, which can then be compared across all cultures involved. Factor analysis is the most frequently employed technique for studying construct equivalence. In the current study both exploratory and confirmatory models could have been used. Given that there is information about the composition of the instrument (on the basis of previous studies), the choice for confirmatory factor analysis may seem obvious. However, the current authors used exploratory factor analysis for a pragmatic reason. The authors had negative experiences with the use of confirmatory models in studies with the MBI-HSS. The main problem in the application of confirmatory models is their fit to the data, which is almost always very bad. It is usually not clear whether the reasons for the poor fit are serious and should lead to a reformulation of the model or are trivial and do not challenge the underlying model.

Exploratory factor analysis was therefore used to examine construct equivalence. A principal components analysis was conducted to determine the number of factors of the MBI-HSS in the total sample. Subsequently, a direct oblimin rotation was used to determine the solution for each race group. Factors obtained in each group were compared (after target rotation). The agreement was evaluated by a factor congruence coefficient, Tucker's phi (Van de Vijver \& Leung, 1997). Values above 0,90 are taken to point to essential agreement between cultural groups, while values above 0,95 point to very good agreement. A high agreement implies that the factor loadings of the lower and higher level are equal up to a multiplying constant. (The latter is needed to accommodate possible differences in eigenvalues of factors for the race groups).

\section{RESULTS}

Because of the composition of the sample, it was decided to conduct the analyses in this study on race groups rather than language groups. Although the best strategy would have been to define cultural groups in terms language, the sample sizes of the language groups were not large enough to satisfy the assumptions of the statistical techniques which were employed.

Firstly, bias analysis was conducted to identify items which show uniform or non-uniform bias for the two race groups. The results of the individual item analysis of variance for the total 22 item MBI-HSS are presented in Table 2.

TABLE 2

ITEM BIAS ANALYSES OF THE MBI-HSS

\begin{tabular}{|c|c|c|}
\hline Item & Uniform Bias & Non-uniform Bias \\
\hline & $p$ & $p$ \\
\hline MBI1 & 0,76 & 0,41 \\
\hline MBI2 & 0,00 & 0,74 \\
\hline MBI3 & 0,08 & 0,27 \\
\hline MBI6 & 0,23 & 0,60 \\
\hline MBI8 & 0,23 & 0,69 \\
\hline MBI13 & 0,01 & 0,56 \\
\hline MBI14 & $0,00^{*}$ & 0,43 \\
\hline MBI16 & 0,00 & 0,15 \\
\hline MBI20 & 0,06 & 0,79 \\
\hline MBI5 & 0,47 & 0,01 \\
\hline MBI10 & 0,05 & 0,45 \\
\hline MBI11 & 0,08 & 0,32 \\
\hline MBI15 & 0,65 & 0,15 \\
\hline MBI22 & 0,01 & 0,02 \\
\hline MBI4 & 0,01 & 0,29 \\
\hline MBI7 & 0,00 & 0,13 \\
\hline MBI9 & 0,03 & 0,01 \\
\hline MBI12 & 0,09 & 0,08 \\
\hline MBI17 & 0,98 & 0,42 \\
\hline MBI18 & 0,00 & 0,61 \\
\hline MBI19 & 0,00 & 0,71 \\
\hline MBI21 & 0,06 & 0,01 \\
\hline
\end{tabular}

${ }^{*} \eta^{2}>0,06$ - Practically significant (medium effect) 
According to Table 2, a significant eta square $\left(\eta^{2}\right)$ value was obtained for item 14 (main effect, medium effect size), which means that this could be regarded as uniformly biased. Item 14 is worded as follows: "I feel I'm working too hard on my job." Therefore, this item was removed from subsequent exploratory factor analyses.

A simple principal components analysis was conducted on the 21 remaining items of the MBI-HSS on the total sample of emergency medical technicians. Analysis of the eigenvalues (larger than 1) and scree plot indicated that four factors could be extracted. However, because previous studies confirmed a threefactor solution and the obtained pattern matrices for a fourfactor solution did not make sense, it was decided to specify three factors. Next, principal component analysis with a direct oblimin rotation was used in carrying out factor analyses per race group. The pattern matrices for Whites and Blacks are reported in Table 3.

TABLE 3

PatTern MATRiX OF MBI-HSS

\begin{tabular}{|c|c|c|c|c|c|c|c|}
\hline \multicolumn{5}{|c|}{ WHITE } & \multicolumn{3}{|c|}{ BLACK } \\
\hline Item & 1 & 2 & 3 & Item & 1 & 2 & 3 \\
\hline MBI1 & 0,77 & $-0,01$ & 0,01 & MBI1 & 0,37 & $-0,20$ & 0,57 \\
\hline MBI2 & 0,58 & 0,03 & 0,06 & MBI2 & 0,17 & $-0,03$ & 0,64 \\
\hline MBI3 & 0,74 & $-0,13$ & $-0,05$ & MBI3 & 0,47 & 0,05 & 0,40 \\
\hline MBI4 & 0,36 & 0,35 & $-0,24$ & MBI4 & $-0,03$ & 0,22 & 0,45 \\
\hline MBI5 & $-0,03$ & $-0,08$ & 0,70 & MBI5 & 0,61 & 0,03 & $-0,09$ \\
\hline MBI6 & 0,24 & $-0,13$ & 0,44 & MBI6 & $-0,06$ & $-0,26$ & 0,63 \\
\hline MBI7 & 0,20 & 0,58 & $-0,16$ & MBI7 & $-0,52$ & 0,40 & 0,46 \\
\hline MBI8 & 0,75 & 0,00 & 0,11 & MBI8 & 0,28 & 0,03 & 0,50 \\
\hline MBI9 & $-0,19$ & 0,61 & 0,05 & MBI9 & $-0,05$ & 0,61 & 0,18 \\
\hline MBI10 & 0,12 & $-0,05$ & 0,62 & MBI10 & 0,62 & 0,05 & $-0,02$ \\
\hline MBI11 & 0,39 & $-0,01$ & 0,27 & MBI11 & 0,61 & 0,16 & 0,31 \\
\hline MBI12 & $-0,56$ & 0,49 & 0,13 & MBI12 & $-0,32$ & 0,50 & 0,20 \\
\hline MBI13 & 0,49 & 0,06 & 0,35 & MBI13 & 0,56 & $-0,07$ & 0,21 \\
\hline MBI15 & $-0,13$ & $-0,09$ & 0,68 & MBI15 & 0,59 & $-0,33$ & $-0,05$ \\
\hline MBI16 & 0,09 & 0,05 & 0,60 & MBI16 & 0,47 & 0,02 & 0,28 \\
\hline MBI17 & 0,06 & 0,79 & $-0,04$ & MBI17 & $-0,02$ & 0,72 & $-0,03$ \\
\hline MBI18 & $-0,14$ & 0,74 & 0,11 & MBI18 & 0,05 & 0,72 & $-0,09$ \\
\hline MBI19 & $-0,06$ & 0,68 & $-0,04$ & MBI19 & 0,25 & 0,73 & $-0,13$ \\
\hline MBI20 & 0,46 & $-0,03$ & 0,45 & MBI20 & 0,75 & 0,05 & $-0,04$ \\
\hline MBI21 & 0,01 & 0,69 & 0,05 & MBI21 & 0,03 & 0,83 & $-0,14$ \\
\hline MBI22 & 0,03 & 0,30 & 0,56 & MBI22 & 0,65 & 0,09 & 0,08 \\
\hline
\end{tabular}

The pattern matrices of the three-factor solutions for Whites and Blacks were then used as input for an exploratory factor analysis with target rotations. The three-factor structure was compared across groups by rotating one solution to the other. After target rotation, the following Tucker's phi coefficients were obtained: a) Factor $1=0,88$; b) Factor $2=0,94$; c) Factor $3=0,70$. Although the Tucker's phi coefficient for Factor 2 compared favourably with the guideline of 0,90 , it is clear that Factors 1 and 3 showed an unacceptable low equivalence for the two race groups.

Inspection of Table 3 revealed that six items were complex and problematic. These items are: a) Item 4 - "I can easily understand how my recipients feel about things."; b) Item $6-$ "Working with people all day is really a strain for me."; c) Item 7 - "I deal very effectively with the problems of my recipients."; d) Item 12 - "I feel very energetic."; e) Item
16 - "Working with people directly puts too much stress on me."; f) Item 22 - "I feel recipients blame me for some of their problems."

The above-mentioned six items either had significant crossloadings on more than one factor, or their loadings on specific factors did not make sense. A closer analysis of the six items showed that all the items had to do with working with people. After removal of the six items, a simple factor analyses was conducted again. The scree plot and eigenvalues showed three factors which explained $50,17 \%$ of the total variance. The pattern matrices for the White and Black groups are reported in Table 4.

TABLE 4

PatTern Matrix OF MBI-HSS

\begin{tabular}{|c|c|c|c|c|c|c|c|}
\hline Item & 1 & 2 & 3 & Item & 1 & 2 & 3 \\
\hline MBI1 & 0,80 & $-0,02$ & $-0,02$ & MBI1 & 0,71 & $-0,17$ & 0,07 \\
\hline MBI2 & 0,64 & 0,04 & $-0,04$ & MBI2 & 0,70 & 0,02 & $-0,02$ \\
\hline MBI3 & 0,77 & $-0,16$ & $-0,08$ & MBI3 & 0,67 & 0,08 & 0,13 \\
\hline MBI5 & $-0,07$ & $-0,04$ & 0,79 & MBI5 & 0,09 & 0,07 & 0,60 \\
\hline MBI8 & 0,80 & 0,00 & $-0,01$ & MBI8 & 0,77 & $-0,02$ & $-0,16$ \\
\hline MBI9 & $-0,12$ & 0,64 & $-0,02$ & MBI9 & 0,24 & 0,61 & $-0,25$ \\
\hline MBI10 & 0,10 & 0,00 & 0,75 & MBI10 & 0,14 & 0,09 & 0,72 \\
\hline MBI11 & 0,35 & 0,02 & 0,42 & MBI11 & 0,63 & 0,17 & 0,32 \\
\hline MBI13 & 0,48 & 0,07 & 0,31 & MBI13 & 0,49 & $-0,11$ & 0,30 \\
\hline MBI15 & $-0,13$ & $-0,04$ & 0,75 & MBI15 & 0,04 & $-0,31$ & 0,67 \\
\hline MBI17 & 0,12 & 0,78 & $-0,07$ & MBI17 & 0,10 & 0,70 & $-0,20$ \\
\hline MBI18 & $-0,04$ & 0,79 & 0,01 & MBI18 & $-0,19$ & 0,74 & 0,18 \\
\hline MBI19 & $-0,03$ & 0,72 & 0,01 & MBI19 & $-0,05$ & 0,75 & 0,26 \\
\hline MBI21 & 0,03 & 0,70 & 0,02 & MBI21 & $-0,07$ & 0,82 & $-0,08$ \\
\hline
\end{tabular}

The three factors were labelled as follows: a) Factor 1: Emotional Exhaustion; b) Factor 2: Personal Accomplishment, and c) Factor 3: Depersonalisation. A target rotation was subsequently carried out on the two pattern matrixes, which resulted in Tucker's phi coefficients of 0,95 (Emotional Exhaustion), 0,92 (Depersonalisation) and 0,96 (Personal Accomplishment). These coefficients can be regarded as highly acceptable.

The descriptive statistics and alpha coefficients of the three factors of the MBI-GS are given in Table 5.

TABLE 5

DESCRIPTIVE STATISTICS, ALPHA COEFFICIENTS AND INTER-ITEM CORRELATIONS OF THE MBI-HSS

\begin{tabular}{lccccc}
\hline Item & Mean & SD & Skewness & Kurtosis & $\alpha$ \\
\hline Emotional Exhaustion & 15,84 & 6,72 & $-0,09$ & $-0,45$ & 0,77 \\
Depersonalisation & 11,03 & 5,55 & 0,01 & $-0,59$ & 0,68 \\
Personal Accomplishment & 20,69 & 5,94 & $-0,50$ & $-0,25$ & 0,78 \\
\hline
\end{tabular}

The information in Table 5 indicates that the three factors of the MBI-HSS are normally distributed. With regards to the internal consistency of the scales, both Emotional Exhaustion and Personal Accomplishment seem to demonstrate acceptable coefficient alphas above the 0,70 guideline provided by Nunnally and Bernstein (1994). In terms of this guideline, the MBI-HSS seems to satisfy the requirements of homogeneity.

These results provide support for Hypotheses 1 and 2. 


\section{DISCUSSION}

The aim of this study was to investigate the psychometric qualities of the MBI-HSS for emergency medical technicians in the Gauteng Province of South Africa, and also to determine construct equivalence and bias for the different race groups in the sample with regards to the burnout construct. Prior to testing for the construct validity and internal consistency of the MBIHSS, construct equivalence and item bias analyses were conducted for the total 22-item questionnaire to determine possible sources of inappropriate comparisons across race groups in this multicultural sample of emergency medical technicians.

Initial analyses showed that the burnout construct was not equivalent for the Black and White groups. Evidence of uniform bias was found for item 14 of the MBI-HSS ("I feel I'm working too hard on my job"). In the case of uniform bias these results point to the fact that for item 14 systematic differences existed between the White and Black groups. This means that even though the same total score on this item could be obtained for members of these race groups, systematic differences in mean scores above (or below) zero could be obtained for individual members of these race groups across all scoring possibilities of this item. The bias which was found on item 14 could have been caused by incidental differences in appropriateness of item content, inadequate item formulation, but also from response characteristics of the sample and administration effects. Alternatively, the limitations due to sample size $(N=318)$, as well as inadequate subgroup sample sizes in terms of the respective race groups could significantly influence the findings. No evidence for non-uniform bias was found.

Based on conceptual and empirical grounds, items 4, 6, 7, 12, 16 and 22 were deleted from the original MBI-HSS, subsequently resulting in a 15 -item scale. This is consistent with the study of Byrne (1993) where item 12 was deleted in a cross-sectional study on the factorial validity of the MBI-HSS in a teacher sample. In her study, Byrne (1993) argued that item 12 ("I feel very energetic") probably does not apply to the teacher sample, accounting for significant cross-loading on two factors. Taken together, the decision to eliminate item 12 is consistent with previous research (Byrne, 1993). The other five items which were omitted from the analysis were all related to working directly with people, which is probably not part of the work situation of emergency medical technicians. These items are formulated as follows:

- Item 4: "I can easily understand how my recipients feel about things." In both race groups this item loaded positively on Emotional Exhaustion, which does not make sense.

- Item 6: "Working with people all day is really a strain for me." While this item loaded correctly on Emotional Exhaustion in the Black group, it loaded on Depersonalisation in the White group.

- Item 7: "I deal very effectively with the problems of my recipients." This item loaded correctly on Personal Accomplishment in the White group, but it had significant loadings on all three factors in the Black group.

- Item 16: "Working with people directly puts too much stress on me." This item loaded on Depersonalisation in both race groups (rather than on Emotional Exhaustion where it was supposed to load).

- Item 22: "I feel recipients blame me for some of their problems." This item loaded on positively on both Personal Accomplishment and Depersonalisation in the White group, while it loaded correctly on Depersonalisation in the Black group.

The three-factor structure of the MBI-HSS was confirmed after removal of six items from the questionnaire. A three-factor structure is consistent with literature findings across various samples, groups and countries (Enzmann et al., 1994; Leiter \& Schaufeli, 1996; Schaufeli \& Enzmann, 1998). Also, reliability analysis confirmed sufficient internal consistency of the subscales. The observed correlations between the subscales were found to be comparable with the values reported in the Maslach Burnout Inventory Manual (Maslach et al., 1996). The correlation between Emotional Exhaustion and Depersonalisation $(r=0,46)$ is somewhat lower than the value of 0,52 reported in the test manual. Correlations of $-0,12$ for Emotional Exhaustion and Personal Accomplishment and -0,15 for Depersonalisation and Personal Accomplishment are slightly lower than the respective values of $-0,22$ and $-0,26$ reported in the test manual. Results from a meta-analytical study by Lee and Ashforth (1996) reported relationships of 0,64 for Emotional Exhaustion and Depersonalisation, $-0,22$ for Emotional Exhaustion and Personal Accomplishment, and -0,34 for Depersonalisation and Personal Accomplishment.

In conclusion, this study could serve as a standard regarding burnout levels in the Gauteng Emergency Services. The threefactor structure of the burnout construct is largely confirmed, as well as the internal consistency of the Emotional Exhaustion, Depersonalisation and Personal Accomplishment scales of the MBI-HSS. Based on the results obtained in the study, it would seem that the MBI-HSS could be regarded as a suitable instrument for measuring burnout in the Emergency Services in Gauteng. The MBI-HSS, therefore paves the way for future burnout research in the Emergency Services in South Africa.

A limitation of this study is its reliance solely on self-report measures. According to Schaufeli et al. (1993), the exclusive use of self-report measures in validation studies increases the likelihood that at least part of the shared variance between measures can be attributed to method variance. Another limitation is the size of the sample, specifically the distribution of language groups and the sampling procedure in the present study, which have significant limitations in terms of the generalisation of the findings applied to the total study population. Future studies could benefit hugely in terms of a stratified random-sample design which would ensure sufficient representation of the different groups in the total population of emergency medical technicians. Also, in terms of the research design, future studies should focus on longitudinal designs where inferences in terms of cause and effect could be made. Future studies conducted in this manner would confirm whether bias and equivalence does indeed exist for the different language groups of the Emergency Services regarding their levels of burnout as measured by the MBI-HSS. Also, the sample should be extended to include the emergency services of other provinces in South Africa in order to standardise the MBI-HSS for the Emergency Services in South Africa.

\section{RECOMMENDATIONS}

According to the results obtained in this study, the use of the MBI-HSS is recommended to assess burnout in Emergency Medical Services in Gauteng. However, items 4, 6, 7, 12, 14, 16 and 22 should be omitted from the questionnaire in the multicultural context.

It is suggested that future research focus on the MBI-HSS in the emergency services in other provinces in South Africa to verify the current findings in terms of inequivalence and bias, as was the case in the present study. Also, although the MBI-HSS was found to be reliable and valid for this sample, other occupational settings should also be investigated in a similar manner. It is recommended that larger samples with a more powerful sampling method be utilised to enable generalisation of the findings to other similar groups. It might also be necessary to translate the MBI-HSS to other languages used in South Africa.

Future research should focus on the usage of both positively and negatively phrased items to measure burnout in emergency medical technicians. Recent research demonstrated that the 
psychometric value of the MBI-GS could be enhanced by including positively phrased items of the Disengagement Scale of the Oldenburg Burnout Inventory (Demerouti, Bakker, Vardakou \& Kantas, 2003). Disengagement, described as distancing oneself from work and experiencing negative attitudes towards work, its content and work in general, was found to be significantly related to Cynicism. Therefore, it is recommended that future studies on burnout in the emergency work setting include the measurement of burnout in a general occupational sense (MBI-GS) with the inclusion of the Depersonalisation scale of the MBI-HSS.

Furthermore, positively phrased items of the Depersonalisation scale of the MBI-HSS should be developed and tested in future studies to determine whether this scale is an artefact of the MBIHSS due to its one-sidedness. In the general sense, the measurement of burnout could be improved psychometrically by including positively phrased items which could even lead to the expansion of the burnout construct beyond Cynicism to a factor measuring mental disengagement from the work object, work content and work in general.

Finally, it cannot be ascertained beyond reasonable doubt that the influence of the "healthy worker effect" (Karasek \& Theorell, 1990) contaminated the current findings, because those seriously affected by the possible prevalence of illness and disabling syndromes, could have left the organisation, leaving the so-called "healthy worker" behind. Furthermore, even if these recipients formed part of the present study, identification could be difficult since no clinical guidelines for the identification of burnout has been developed for the South African labour force in different occupational settings. This is furthermore compounded by the nonprobability sampling procedure used in the present study. Future research should therefore focus on the development of clinical guidelines in terms of burnout in various occupational settings to enable comparison and identification across occupations according to national guidelines.

\section{REFERENCES}

Basson, M. \& Rothmann, S. (2002, March). Sense of coherence, coping and burnout of pharmacists. Poster presented at the $1^{\text {st }}$ South African Burnout Conference, Potchefstroom, South Africa.

Bentler, P.M. (1992). On the fit of models to covariances and methodology to the bulletin. Psychological Bulletin, 112, 400404.

Boles, J.S., Dean, D.H., Ricks, J.M., Short, J.C. \& Wang, G. (2000). The dimensionality of the Maslach Burnout Inventory across small business owners and educators. Journal of Vocational Behavior, 56, 12-34.

Brill, P.L. (1984). The need for an operational definition of burnout. Family and Community Health, 6, 12-24.

Brookings, J.B., Bolton, B., Brown, C.E. \& McEvoy, A. (1985). Selfreported job burnout among female human service professionals. Journal of Occupational Behavior, 6, 143-150.

Byrne, B.M. (1991). The Maslach Burnout Inventory: Validating factorial structure and invariance across intermediate, secondary, and university educators. Multivariate Behavioral Research, 26, 477-499.

Byrne, B.M. (1994). Burnout: Testing for the validity, replication and invariance of causal structure across elementary, intermediate, and secondary teachers. American Educational Research Journal, 31, 645-673.

Cleary, T.A. \& Hilton, T.L. (1968). An investigation of item bias. Educational and Psychological Measurement, 28, 61-75.

Cordes, C.L. \& Dougherty, T.W. (1993). A review and an integration of research on job burnout. Academy of Management Review, 18, 621-656.

Demerouti, E., Bakker, A.B., Vardakou, I. \& Kantas, A. (2003). The convergent validity of two burnout instruments: A multitrait-multimethod analysis. European Journal of Psychological Assessment, 19 (1), 12-23.
Enzmann, D., Schaufeli, W.B. \& Girault, N. (1994). The validity of the Maslach Burnout Inventory in three national samples. In L. Bennet, D. Miller \& M. Ross (Eds.), Health workers and AIDS: Research, interventions and current issues (pp. 131-150). London: Harwood.

Firth, H., McIntee, J., McKeown, P. \& Britton, P.G. (1985). Maslach Burnout Inventory: Factor structure and norms for British nursing staff. Psychological Reports, 57, 147-150.

Frank, J.R. \& Ovens, H. (2002). Shiftwork and emergency medical practice. The Journal of the Canadian Association of Emergency Physicians, 4, 421-428.

Friesen, D., Prokop, C.M. \& Sarros, J.C. (1988). Why teachers burn out. Educational Research Quarterly, 12, 9-19.

Golembiewski, R.T. \& Munzenrider, R. (1981). Efficacy of three versions of one burn-out measure: MBI as total score, subscale scores, or phrases? Journal of Health and Human Resources Administration, 7, 228-246.

Green, D. \& Walkey, F.H. (1988). Confirmation of the threefactor structure of the Maslach Burnout Inventory. Educational and Psychological Measurement, 48, 579-585.

Iwanicki, E.F. \& Schwab, R.L. (1981). A cross validation study of the Maslach Burnout Inventory. Educational and Psychological Measurement, 41, 1167-1174.

Jackson, S.E. \& Maslach, C. (1982). After-effects of job-related stress: Families as victims. Journal of Occupational Behavior, 3, 63-67.

Jackson, S.E., Schwab, R.L. \& Schuler, R.S. (1986). Toward an understanding of the burnout phenomenon. Journal of Applied Psychology, 71, 630-640.

Karasek, R. \& Theorell, T. (1990). Healthy work: Stress, productivity and the reconstruction of working life. New York: Basic Books.

Koeske, G.F. \& Koeske, R.D. (1993). Construct validity of the Maslach Burnout Inventory: A critical review and conceptualisation. Journal of Applied Behavioral Science, 25, $131-132$

Kowalski, K.M. \& Vaught, C. (2001). The safety and health of emergency workers. Journal of Contingencies \& Crisis Management, 9, 138-143.

Lee, R.T. \& Ashforth, B.E. (1996). A meta-analytical examination of the correlates of the three dimensions of job burnout. Journal of Applied Psychology, 81, 123-133.

Leiter, M.P. \& Durup, J. (1994). The discriminant validity of burnout and depression: A confirmatory factor analytic study. Anxiety, Stress and Coping, 7, 357-373.

Leiter, M.P. \& Durup, J. (1996). Work, home, and in-between: A longitudinal study of spill-over. Journal of Applied Behavioral Science, 32, 29-47.

Levert, T., Lucas, M. \& Ortlepp, K. (2000). Burnout in psychiatric nurses: Contributions of the work environment and a Sense of Coherence. South African Journal of Psychology, 30, 36-43.

Maslach, C. (1982). Burnout: The cost of caring. Englewood Cliffs, NJ: Prentice Hall.

Maslach, C. \& Jackson, S.E. (1981). The measurement of experienced burnout. Journal of Occupational Behavior, 2, 99-113.

Maslach, C. \& Jackson, S.E. (1984). Burnout in organizational settings. In O.S. Oskamp (Ed.), Applied social psychology annual: Volume 5 -Applications in organizational settings. (pp. 133-153). Beverly Hills, CA: Sage.

Maslach, C. \& Jackson, S.E. (1986). Maslach Burnout Inventory (2nd ed.). Palo Alto, CA: Consulting Psychologist Press.

Maslach, C., Jackson, S.E. \& Leiter, M. (1996). Maslach Burnout Inventory: Manual (3rd ed.). Palo Alto, CA: Consulting Psychologists Press.

Maslach, C., Jackson, S.E. \& Leiter. M. (1997). Maslach Burnout Inventory: Third Edition. In C.P. Zalaquett \& R.J. Wood (Eds.), Evaluating stress: A handbook of resources (pp. 191-218). London: Scarecrow Press.

Mellenbergh, G.J. (1982). Contingency table models for assessing item bias. Journal of Educational Statistics, 7, 105-118.

Muthén, B.O. (1991). Multilevel factor analysis of class and student achievement components. Journal of Educational Measurement, 28, 338-354. 
Muthén, B.O. (1994). Multilevel covariance structure analysis. Sociological Methods \& Research, 22, 376-398.

Nunnally, J.C. \& Bernstein, I.H. (1994). Psychometric theory (3 ${ }^{\text {rd }}$ ed.). New York: McGraw-Hill.

Phipps, L. (1988). Stress among doctors and nurses in the emergency department of a general hospital. Canadian Medical Association Journal, 139, 375-376.

Rosse, J.G., Boss, R.W., Johnson, A.E. \& Crown, D.F. (1991). Conceptualizing the role of self-esteem in the burnout process. Group and Organization Studies, 16, 197-204.

Rothmann, S. (2003). Burnout and engagement: A South African perspective. South African Journal of Industrial Psychology, 29 (4), 16-25.

Schaufeli, W.B., Bakker, A.B., Hoogduin, K., Schaap, C. \& Kladler, A. (2001). On the clinical validity of the Maslach Burnout Inventory and the Burnout Measure. Psychology and Health, 16, 565-582.

Schaufeli, W.B. \& Buunk, B.P. (2002) Burnout: An overview of 25 years of research and theorizing. M.J. Schabracq, J.A.M. Winnubst, C.L. Cooper (Eds.), Handbook of work and health psychology (pp. 383-425). Chichester: Wiley.

Schaufeli, W.B. \& Enzmann, D. (1998). The burnout companion to study and practice: A critical analysis. London: Taylor \& Francis.

Schaufeli, W.B., Enzmann, D. \& Girault, N. (1993). Measurement of burnout: A review. In W.B. Schaufeli, C. Maslach \& T. Marek (Eds.), Professional burnout: Recent developments in theory and research (pp. 199-215). Washington, DC: Taylor \& Francis.

Schaufeli, W.B., Martinez, I., Pinto, A.M., Salanova, M. \& Bakker, A.B. (2002). Burnout and engagement in university students: A cross national study. Journal of Cross-Cultural Psychology, 33, 464-481.
Schaufeli, W.B. \& Van Dierendonck, D. (1995). A cautionary note about the cross-national and clinical validity of cut-off points for the Maslach Burnout Inventory. Psychological Reports, 76, 1083-1090.

Schwab, R.L. \& Iwanicki, E.F. (1982). Perceived role conflict, role ambiguity, and teacher burnout. Educational Administration Quarterly, 18, 60-74.

Shaughnessy, J.J. \& Zechmeister, E.B. (1997). Research methods in psychology (4th ed.). New York: McGraw-Hill.

SPSS Inc. (2003). SPSS 12.0 for Windows. Chicago, IL: Author.

Storm, K. \& Rothmann, S. (2003). A psychometric analysis of the Maslach Burnout Inventory - General Survey in the South African Police Service. South African Journal of Psychology, 33, 219-226.

Van de Vijver, F. \& Leung, K. (1997). Methods and data analysis for cross-cultural research. Thousand Oaks, CA: Sage.

Van de Vijver, F. \& Poortinga, Y.H. (1994). Methodological issues in cross-cultural studies on parental rearing behaviour and psychopathology. In C. Perris, W.A. Arrindell \& M. Eisemann (Eds.), Parental rearing and psychopathology (pp. 173-197). Chichester, UK: Wiley.

Vettor, S.M. \& Kosinski, F.A. (2000). Work-stress and burnout in emergency medical technicians and the use of early recollections. Journal of Employment Counseling, 37, 216-228.

Whitley, T.W., Gallery, M.E., Allison, E.J. \& Revicki, D.A. (1989). Factors associated with stress among emergency medical residents. Annual Emergency Medical, 18, 1157-1161.

Young, K.M. \& Cooper, C.L. (1995). Occupational stress in the ambulance service: A diagnostic study. Journal of Managerial Psychology, 10 (3), 29-36. 